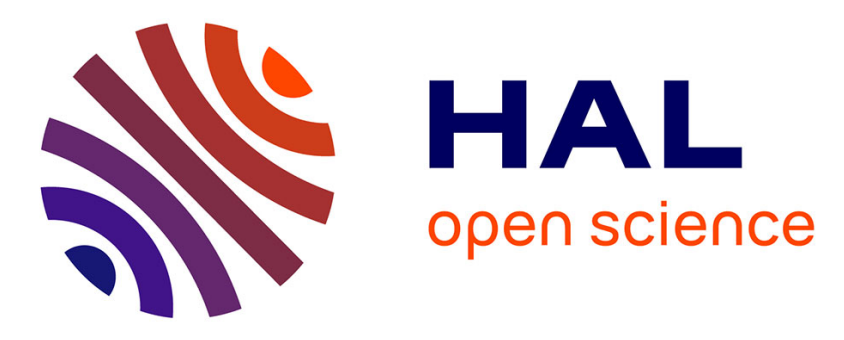

\title{
Electrokinetic elucidation of the interactions between persistent luminescent nanoprobes and the binary apolipoprotein-E/albumin protein system
}

Gonzalo Ramírez García, Fanny d’Orlyé, Cyrille Richard, Nathalie Mignet, Anne Varenne

\section{To cite this version:}

Gonzalo Ramírez García, Fanny d'Orlyé, Cyrille Richard, Nathalie Mignet, Anne Varenne. Electrokinetic elucidation of the interactions between persistent luminescent nanoprobes and the binary apolipoprotein-E/albumin protein system. Analyst, 2021, 146 (17), pp.5245-5254. 10.1039/d1an00781e . hal-03482252

\section{HAL Id: hal-03482252 https://hal.science/hal-03482252}

Submitted on 15 Dec 2021

HAL is a multi-disciplinary open access archive for the deposit and dissemination of scientific research documents, whether they are published or not. The documents may come from teaching and research institutions in France or abroad, or from public or private research centers.
L'archive ouverte pluridisciplinaire HAL, est destinée au dépôt et à la diffusion de documents scientifiques de niveau recherche, publiés ou non, émanant des établissements d'enseignement et de recherche français ou étrangers, des laboratoires publics ou privés. 
Received 00th May 2021, Accepted 00th January 20xx DOI: $10.1039 / x 0 x \times 00000 x$

\title{
Electrokinetic elucidation of the interactions between persistent luminescence nanoprobes and the binary Apolipoprotein- $E$ /Albumin protein system
}

Gonzalo Ramírez García, ${ }^{\mathrm{a}, \mathrm{b}, \mathrm{c}}$ Fanny d'Orlyé, ${ }^{\mathrm{b}}$ Cyrille Richard, ${ }^{\mathrm{c}}$ Nathalie Mignet, ${ }^{\mathrm{c}}$ and Anne Varenne ${ }^{\mathrm{b}, *}$

\begin{abstract}
The affinity between functional nanoparticles (NPs) and proteins could determine the efficacy of nanoprobes, nanosensors, nanocarriers, and many other devices for biomedical applications. Thereby, it is necessary to develop analytical strategies to accurately evaluate the magnitude of these protein-corona interactions in physiological media. In this work, different electrokinetic strategies were implemented to accurately determine the interactions between PEGylated $\mathrm{ZnGa}_{1.995} \mathrm{Cr}_{0.005} \mathrm{O}_{4}$ persistent luminescence NPs (ZGO-PEG) and two important serum proteins: human serum albumin (HSA), the most abundant serum protein, and apolipoprotein-E (ApoE), associated with the active transport of NPs through the blood-brain barrier. Firstly, the injection of ZGO-PEG in a background electrolyte (BGE) containing individual proteins allowed an affinity study to separately characterize each NPs-protein system. Then, the same procedure was applied in a buffer containing a mixture of the two proteins at different molar ratios. Finally, the NPs were pre-incubated with one protein and thereafter electrokinetically separated in a BGE containing the second protein. These analytical strategies revealed the mechanisms (comparative, cooperative or competitive systems) and magnitude of their interactions, resulting in all cases in notably higher affinity and stability between the ZGO-PEG and the ApoE $\left(\mathrm{Ka}=1.96 \pm 0.25 \times 10^{10} \mathrm{M}^{-1}\right)$ compared to HSA $\left(\mathrm{Ka}=4.60 \pm 0.41 \times 10^{6} \mathrm{M}^{-1}\right)$. For the first time, the inter-protein ApoE/HSA interactions with ZGO-PEG were also demonstrated, highlighting the formation of a ternary ZGO-PEG/ApoE/HSA nanocomplex. These results open the way for a deeper understanding of the protein corona formation, and the development of versatile optical imaging applications for the ZGO-PEG and other systemically delivered nanoprobes ideally vectorized to the brain.
\end{abstract}

\section{Introduction}

In the current context of nanomedicine, one of the most representative challenges is to successfully target therapeutic NPs to cells or organs of interest. ${ }^{1}$ However, some constraints such as their detection by the immune system, ${ }^{2}$ degradation, ${ }^{3}$ bioaccumulation by organs, ${ }^{4}$ or the presence of physiological barriers limit their adequate targeting and function. ${ }^{5}$ For instance,

a. Centro de Física Aplicada y Tecnología Avanzada, Universidad Naciona

Autónoma de México. 3001, Blvd. Juriquilla, 76230, Querétaro, México.

b. Chimie ParisTech, PSL University, CNRS 2027, Institute of Chemistry for Life and Health Sciences, 75005, Paris, France.

c. Université de Paris, CNRS, INSERM, UTCBS: Chemical and Biological Technologies

for Health, Faculté de Pharmacie, 75006, Paris, France.

* Corresponding author. E-mail: anne.varenne@chimieparistech.psl.eu the blood-brain barrier (BBB) is one of the most impermeable physiological barriers in the organism, and it enables protection of the brain from the peripheral circulation and toxic substances but restricts the transport of many therapeutically relevant nanodrugs. ${ }^{6}$ In this context, some early studies have shown a clear correlation between the apolipoprotein-E (ApoE) adsorption onto the NPs surface and the passage through the BBB, and different kinds of nanocarriers have been successfully used for the transport of drugs to the brain. ${ }^{7,8}$ However, once in vivo, the NPs interacts with plasma proteins and other biomolecules, forming new complexes and dynamic entities in which an active exchange of proteins from solution to the NP surface occurs. ${ }^{1}$ Thereby, the rapid formation of a protein corona could critically affect the interactions of the NPs 
with living systems, and thereby, their distribution and therapeutic

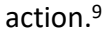

The use of effective imaging agents will help to clarify the precise mechanism for NPs distribution and interactions in the body, and to diagnose diseases in earlier stages. As reported in our group, the $\mathrm{ZnGa}_{1.995} \mathrm{Cr}_{0.005} \mathrm{O}_{4}$ persistent luminescence NPs (ZGO-NPs) represent a new generation of optical nanoprobes, whose persistent luminescence can be activated before administration on live systems, as well as in vivo through living tissues. ${ }^{10}$ This in situ reactivation in the therapeutic window results in the ability to make observations of the probe without any time constraints, opening new perspectives for a great variety of diagnosis applications. For instance, the ability of these PEGylated ZGO-NPs for in vivo passive tumor targeting without long-term toxic effects has been demonstrated.11,12 These advantages conduct to consider the adsorption of plasma proteins at the surfaces of these NPs, in order to program the final fate of the ZGO-NPs or other functional NPs into the organism.

A few recent publications showed different ways by which capillary electrophoresis (CE) can be applied to determine qualitative and quantitative information about the interactions between NPs and various types of proteins. ${ }^{13-16}$ Different approaches have been used for this purpose, principally the affinity CE mode (ACE), providing the determination of binding constants and kinetics, stoichiometry and cooperativity. ${ }^{13,15-18}$ The application of the Hummel-Dreyer CE (HDCE) mode for evaluation of the interactions between the ZGOPEG NPs and bovine serum albumin (BSA) was also demonstrated previously in our group. ${ }^{14}$ Furthermore, the capillary zone electrophoresis (CZE) has shown to be an important tool in the analysis of the ApoE protein involvement in the translocation of nanocarriers through the $\mathrm{BBB}, 8,19,20$ and it has been also used as a complementary method to study the absorption of specific peptides onto PEGylated nanoparticles. ${ }^{21,22}$

In the present work, the affinity between ZGO-PEG NPs and a binary system of proteins (HSA and ApoE) was systematically evaluated through an integral set of tests including comparative, cooperative, and competitive models in a capillary electrophoresis system. The presented methodology allows the rapid and versatile determination of both stable or short-lived NPs-protein nanocomplexes with fast or slow association/dissociation rates in physiologically relevant conditions. Since the protein adsorption by NPs provides them new identity and properties, this strategy can enhance the development of novel biomedical applications of the NPs, such as biosensors, optical imaging, or targeted therapy. Furthermore, the possibility of modifying the ZGO-PEG surface with ApoE by a simple and fast incubation step is discussed in this work, in order to envision the potential application of the ZGO-PEG/ApoE nanocomplex for the development of optical imaging agents to detect brain pathologies.

\section{Experimental}

The detailed experimental protocols used for the synthesis, functionalization, and characterization of the ZGO-PEG are presented in the Supporting Information section.

\section{Analytical procedures for evaluation of interactions between nanoparticles and proteins by CE}

Initially, the proteins were individually analyzed by means of CZE. Injections of $1.44 \mu \mathrm{M}$ HSA or ApoE suspensions were performed hydrodynamically by applying 20 mbar at the capillary inlet for $10 \mathrm{~s}$ $(10 \mathrm{~nL})$. The background electrolyte (BGE) was $30 \mathrm{mM} \mathrm{ACB}(\mathrm{pH}$ 7.4). The separation was performed under a voltage of $14 \mathrm{kV}$, and the signals obtained with a UV-Vis detector $(\lambda=200 \mathrm{~nm})$. The molar extinction coefficients of the proteins were determined at $200 \mathrm{~nm}$ under these experimental conditions according to the Lambert-Beer law, and considering the equation $\mathrm{A}=\varepsilon \mathrm{CL}$, (where $\mathrm{A}=$ absorbance, $\varepsilon=$ molar extinction coefficient, $C=$ molar concentration and $L$ the light pathway). Viscosity and conductivity measurements of the BGE containing mixtures of proteins at different ratios were carried out according to a previously reported CE protocol. ${ }^{23}$

To evaluate the interactions between ZGO-PEG and the proteins, three different CE approaches were performed: a) comparative interactions of NPs with individual HSA or ApoE proteins at different concentrations under the same analytical conditions, b) cooperative interactions of NPs with a binary system of proteins mixed at different ratios, and c) incubation of the ZGO-PEG NPs with one of the proteins during 15 minutes, and thereafter the analysis of their competitive interactions in a separation medium containing the second protein, and vice versa.

The overall method used for the different experiments performed in this section consists of five principal steps: i) equilibrium of the capillary, consisting of successive flushes with $0.1 \mathrm{M} \mathrm{NaOH}$ (2 min), $\mathrm{H}_{2} \mathrm{O}$ (2 min) and ACB (2 min) at corresponding ionic strength (15 or 
$30 \mathrm{mM}$ ). ii) capillary preconditioning, in which the BGE was flushed for 3 minutes. For comparative or competitive interactions, the BGE consisted of HSA or ApoE at varied concentrations dispersed in 30 $\mathrm{mM}$ ACB solutions. For cooperative interactions, the BGE was a mixture of HSA and ApoE at different ratios dispersed in $15 \mathrm{mM}$ ACB solutions. iii) sample injection, in which $0.2 \mathrm{mg} / \mathrm{mL}$ ZGO-PEG or ZGO-PEG pre-incubated with one of the proteins were hydrodynamically injected (applying 20 mbar at the capillary inlet for 10s) for comparative or competitive interactions, respectively. In the case of the cooperative interactions, ZGO-PEG samples were injected only for $5 \mathrm{~s}$ at the same pressure in a capillary filled with protein mixtures. The electropherogram of $0.001 \%$ DMF in BGE was obtained after each sample (4 replicates) to control the electroosmotic flow. iv) electrokinetic separation, consisting of the application of a voltage ranging from 12 to $14 \mathrm{kV}$ (see the precise value in the footnote of every figure). The signal registration was made with a UV-detector at $\lambda=200 \mathrm{~nm}$. v) analysis of interactions, based in two different approaches for numerical data interpretation. First, the Hummel-Dreyer method (HD-CE) was applied in the cases in which no shifts in electrophoretic mobility of the involved species (protein, NPs, or their complex) were detected upon protein concentration variation. There, the injection of $A C B$ solutions under the same analytical conditions was used for external calibration, and the peak areas were monitored to obtain the portion of protein bounded to NPs and the derived analytical parameters. The Affinity CE (ACE) was applied for interpretation of the interactions in which considerable shifts in the electrophoretic mobilities of the nanocomplex formed between ZGO-NPs and the proteins were detected after variation of the protein concentrations.

A Model G7100A CE system from Agilent Technologies equipped with a UV-Vis absorbance detector was used for the analysis. Separations were carried out with a fused silica capillary $[37 \mathrm{~cm}$ (effective length $28.5 \mathrm{~cm}$ ) x $50 \mu \mathrm{m}$ I.D., purchased from Polymicro Technologies (Phoenix, USA)] activated by successive flushes (925mbar) with $1.0 \mathrm{M} \mathrm{NaOH}$ (15 min), 0.1 M NaOH (15 min) and $\mathrm{H}_{2} \mathrm{O}(5 \mathrm{~min})$, respectively.

\section{Results and discussion}

ZGO-NPs were synthesized, and afterward a three steps functionalization sequence was set up to obtain the PEG-modified
NPs (Figure $1 \mathrm{~A}$ ). The PEG chains are intended to reduce the NP interactions with various proteins and biological elements, to reduce their detection by the mononuclear phagocyte system, ${ }^{24}$ and thereby, to improve their biodistribution and applications as optical imaging tool. An average solid diameter of $30 \mathrm{~nm}$ with semispherical shape was observed via TEM (Figure 1B), while the success of PEGylation was confirmed by FTIR spectra (Figure 1C). The hydrodynamic diameter of the PEGylated NPs was $177.6 \pm 7.6 \mathrm{~nm}$ in $30 \mathrm{mM}$ ammonium carbonate (ACB) at $\mathrm{pH} 7.4$, with a polydispersity index smaller than 0.1 , demonstrating that monodisperse ZGO-NP formulations were obtained (Figure 1D). The zeta potential was almost neutral, with a value of $-1.7 \pm 0.6 \mathrm{mV}$. This is attributed to the long chains of PEG (5kD) screening the charge of the NPs. A detailed description of the physicochemical characterization of these NPs in physiologically relevant media is presented in the Supporting Information section, indicating a preserved colloidal stability of the ZGO-PEG above all the range of conditions used in the present work.

\section{Interactions between ZGO-PEG and individual proteins: affinity electrokinetic studies with HSA or ApoE suspensions}

In a first step, the HSA and ApoE proteins were electrokinetically characterized before studying their interactions with ZGO-PEG so as to discard any possible interference (Figure 2). The electrophoretic mobilities of HSA and ApoE in $30 \mathrm{mM} \mathrm{ACB}(\mathrm{pH}$ 7.4) are both negative as obtained by means of CZE $\left(-1.52 \pm 0.12 \times 10^{-4}\right.$ and $-1.65 \pm$ $0.09 \times 10^{-4} \mathrm{~cm}^{2} \mathrm{~V}^{-1} \mathrm{~s}^{-1}$, respectively), which is in agreement with their isoelectric points (4.7 and 5.5 respectively). As evidenced in the electropherograms, the absorbance intensity of the proteins is however significantly different. According to the Lambert-Beer law, the molar extinction coefficients $(\varepsilon)$ were determined at $200 \mathrm{~nm}$ with the CE apparatus under the experimental conditions (30 mM $\mathrm{ACB}, \mathrm{pH}$ 7.4), resulting in $\varepsilon=1.48 \times 10^{6} \pm 0.03 \mathrm{~cm}^{-1} \mathrm{M}^{-1}$ and $\varepsilon=1.17$ $\times 10^{5} \pm 0.02 \mathrm{~cm}^{-1} \mathrm{M}^{-1}$ for $\mathrm{HSA}$ and $\mathrm{ApoE}$, respectively. At this wavelength, absorbance arises primarily from the peptide backbone, independently of the protein sequence. ${ }^{25}$ The HSA is notably bigger (M.W. 66500 Da, 585 amino acids) than ApoE (M.W. $34200 \mathrm{Da}, 299$ amino acids). ${ }^{26}$ Thereby, the difference in the observed $\varepsilon$ values could be related to the considerable variation in the size of the analyzed proteins. 
A)
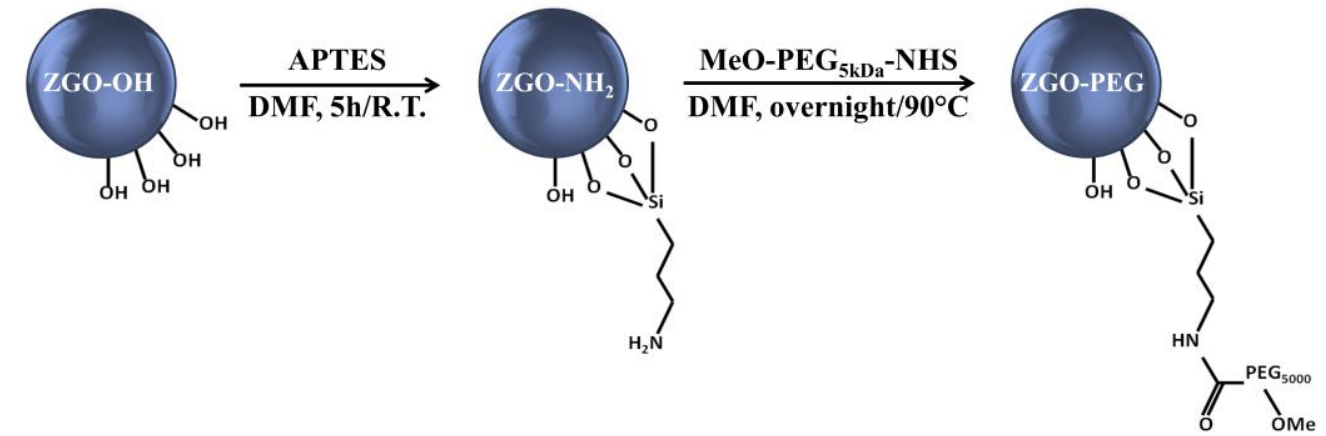

B)

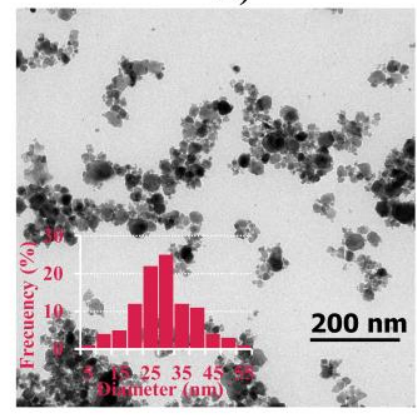

C)

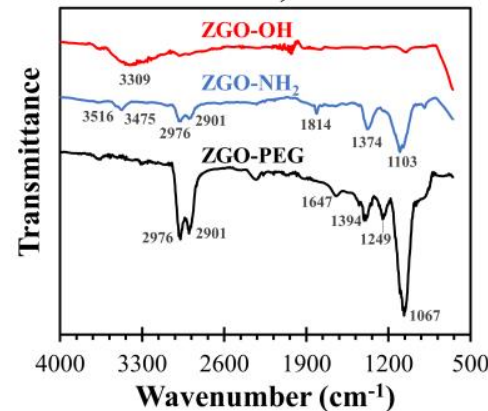

D)

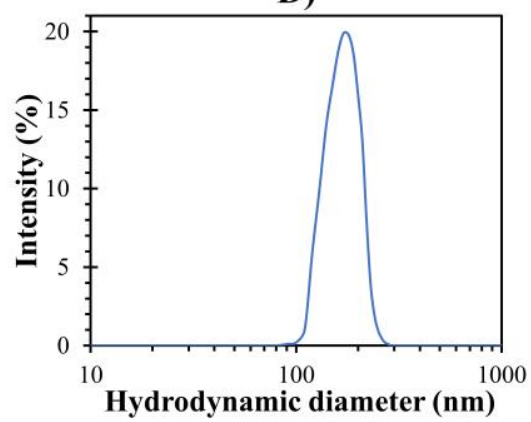

Figure 1. A) Schematic representation of the $\mathrm{ZnGa}_{1.995} \mathrm{Cr}_{0.005} \mathrm{O}_{4}$ surface functionalization sequence, B) TEM image of the ZGO-PEG NPs. The inset shows the corresponding histogram for size distribution. C) FTIR-ATR spectra of the intermediary functionalized ZGO-NPs. D) DLS profile of ZGO-PEG in pH 7.4 ACB $30 \mathrm{mM}$.

While the HSA is easily observed, the analysis of the ApoE is limited to concentrations higher than $1.44 \mu \mathrm{mol} \mathrm{L}^{-1}$.

In a second step, the ZGO-PEG NPs were electrokinetically separated in $30 \mathrm{mM}$ ACB (Figures $3 \mathrm{Ab}$ and $3 \mathrm{Bb}$ ). The low surface charge density of the ZGO-PEG, and thereafter the quite neutral electrophoretic mobility is due to the presence of the $5 \mathrm{kD}$ PEG layer. When the ZGO-NP-protein interactions are analyzed, at least two possible systems can be expected: i) stable NPs-protein complexes or ii) short-lived complexes with fast association/dissociation rates. The choice of the adequate electrokinetic separation mode for evaluation of the NP-protein interactions is therefore crucial. In the present section, an "affinity" methodology was implemented, consisting in the electrokinetic separation of the ZGO-PEG NPS in a BGE containing individual proteins at different concentrations. This methodology resulted in two markedly different electrophoretic behaviors when HSA or ApoE were analyzed under the same analytical conditions (Figure 3).
Considering the colloidal stability of the ZGO-PEG NPs, the variations in electrophoretic profiles can be exclusively associated to the NPs/proteins or inter-protein interactions.

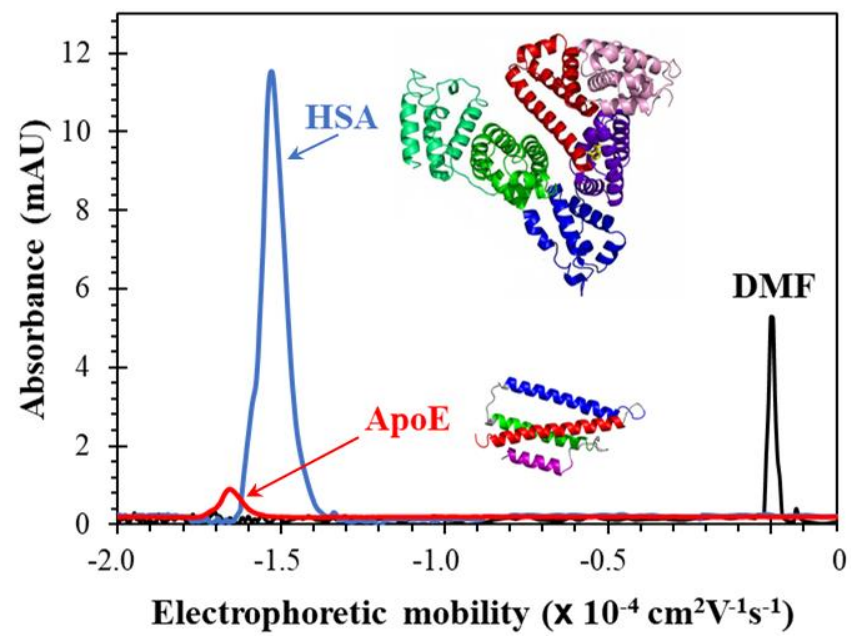

Figure 2. Electropherograms of $H S A$ and $\operatorname{ApoE}(1.44 \mu \mathrm{M})$ in a capillary pre-conditioned with $30 \mathrm{mM} \mathrm{ACB}(\mathrm{pH}$ 7.4). The separation was performed with $E=14 \mathrm{kV}$, and the signals obtained with a UVVis detector $(\lambda=200 \mathrm{~nm})$. 


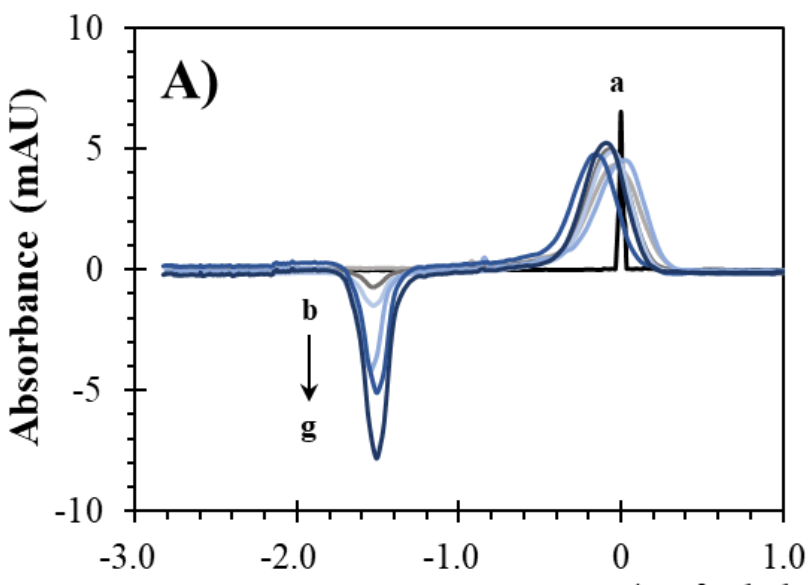

Electrophoretic mobility $\left(\times 10^{-4} \mathrm{~cm}^{2} \mathrm{~V}^{-1} \mathrm{~s}^{-1}\right)$

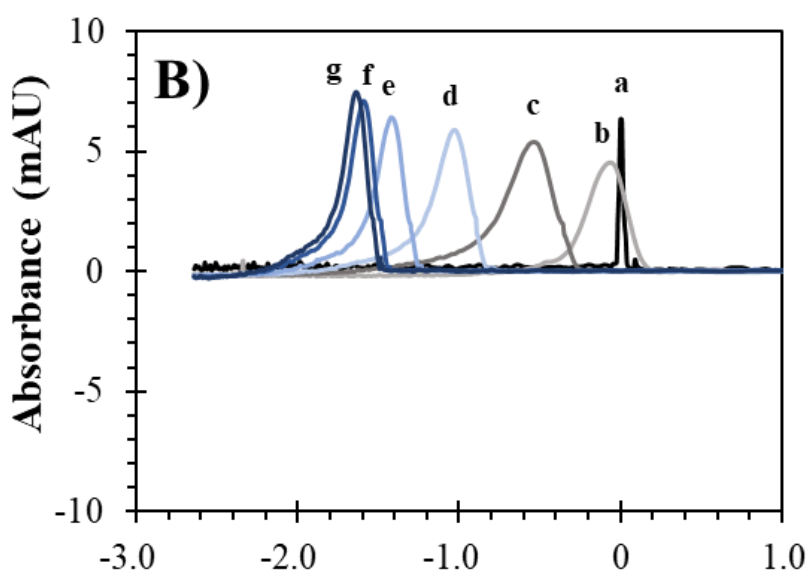

Electrophoretic mobility $\left(\times 10^{-4} \mathrm{~cm}^{2} \mathrm{~V}^{-1} \mathrm{~s}^{-1}\right)$

Figure 3. Representative electropherograms for the comparative analysis of interactions between ZGO-PEG and A) HSA or B) ApoE, in a capillary pre-conditioned with varied protein concentrations in ACB $(\mathrm{pH} \mathrm{7.4)}$ at $30 \mathrm{mM}$ ionic strength. The separation was performed with $\mathrm{E}=14 \mathrm{kV}$, and the signals obtained with a UV-Vis detector $(\lambda=200 \mathrm{~nm})$. Peak identification: a) DMF, b) 0, c) 0.12, d) 0.24 , e) 0.72 , f) 0.96, g) $1.44 \mu \mathrm{M}$ of the corresponding protein.

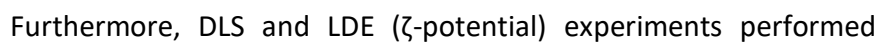
under similar conditions did not provide exploitable results. Indeed, the separation ability of CE is paramount to detect small changes in the magnitude of association parameters, and thereby, for the determination of the interactions.

In the case of interactions with HSA (Figure $3 \mathrm{~A}$ ), two peaks were evidenced when HSA was present in the BGE. A "negative peak" (vacancy peak) appears at a constant electrophoretic mobility corresponding to the one of HSA, and is due to a local depletion of HSA in the BGE originating from the ZGO-PEG/HSA complex formation. A "positive peak" at a nearly zero electrophoretic mobility corresponds to the ZGO-NPs or their nanocomplexes with HSA. A gradual increase in the vacancy peak area of HSA was evidenced upon increase of its concentration in the BGE, while no significant shift in the electrophoretic mobility or variation on the shape of the "positive peak" was observed. This electrophoretic evolution of ZGO-PEG as a function of the HSA concentration (from 0 to $1.44 \mu \mathrm{M}$ ) in $30 \mathrm{mM} \mathrm{ACB}$, corresponds to the Hummel-Dreyer CE (HDCE) mode.

On the other hand, only one positive peak was evidenced with a gradual shift in electrophoretic mobility upon the increase of ApoE concentration in the BGE (Figure $3 \mathrm{~B}$ ). The lower molar extinction coefficient of ApoE (one order of magnitude compared to the HSA, as determined above) can explain the absence of ApoE signal in the experienced concentrations. The variations in the electrophoretic mobility of the positive peak indicate the formation of a stable nanocomplex between ZGO-PEG and ApoE. Since ApoE is negatively charged under the experimental conditions, the interaction of ZGOPEG with ApoE induces a gradual increase (in negative value) of the electrophoretic mobility. This evolution corresponds to the affinity CE (ACE) mode, being the first evidence of the stable NP-ApoE interaction.

The significant differences in the electrophoretic profiles obtained for the evaluation of the interactions between ZGO-PEG and HSA or ApoE could be explained in terms of interaction strengths and kinetics. The electrophoretic mobility shifts derived from the interactions with ApoE demonstrate a fast binding with $A p o E$, and a high stability of the ZGO-PEG/ApoE complex. The electrophoretic mobility shifts towards more negative values for higher protein concentrations are due to the increase of charged groups provided by the ApoE molecules at the surface of the PEGylated NPs. On the other hand, the absence of variations in the shape and electrophoretic mobility of the ZGO-PEG upon interaction with HSA, and the presence of a vacancy peak of the protein, could indicate a faster association/dissociation kinetics compared to ApoE. The protein size could strongly impact the affinity with the NPs, since a smaller size can lead to better spatial distribution and thus stronger interactions. This CE methodology is then a convenient analytical tool for comparison of the interaction dynamics between ZGO-PEG and both HSA or ApoE proteins.

Since the electrophoretic profiles observed for ZGO-PEG interactions with both HSA and ApoE proteins present significant differences, two numerical approaches have been applied to 
determine the ZGO-PEG/protein binding constants and association parameters. A detailed description of the equations and models applied in this work is developed in the Supporting Information section. For the ZGO-PEG/HSA nanocomplex in $30 \mathrm{mM}$ ACB $(\mathrm{pH}$ 7.4), the model fitting parameters according to the HDCE mode leads to a $K_{a}=4.60 \pm 0.41 \times 10^{6} \mathrm{M}^{-1}$ and a number of binding sites $\mathrm{n}=$ $4.35 \pm 0.24$. Otherwise, the ACE mode leads to a $K_{a}=1.96 \pm 0.25 \times$ $10^{10} \mathrm{M}^{-1}$ and a slightly cooperative association $\left(n_{H}=1.47 \pm 0.07\right)$ for the ZGO-PEG/ApoE nanoconjugate. The stronger ZGO-PEG/ApoE binding compared to ZGO-PEG/HSA was evidenced by these calculations, resulting in a difference of four orders of magnitude. In general, proteins could have affinity with other substances through several interactions like $\mathrm{H}$-bonds, pi-stacking, and electrostatic attractions, which could be responsible for the interactions observed in this work. The qualitative adsorption of ApoE onto other PEGylated NPs has also been verified by 2D-polyacrylamide gel electrophoresis (2D-PAGE). ${ }^{27,28}$ Thereby, the PEGylation does not exclude the NPs to interact with circulating proteins, for instance those related to the immune system or mononuclear phagocyte system. ${ }^{29}$ This stronger affinity between ZGO-PEG and ApoE could be related to the high-affinity for the lipid-binding region in the C-terminal domain (amino acids 244-272) present in this protein. This region appears to be linked to the primary ApoE functions, such as ligands binding. ${ }^{30}$ The smaller size of ApoE compared to HSA could also enhance the interactions by improving their spatial distribution and orientation at the NP surface.

\section{Interactions between ZGO-PEG and a mixture of the HSA and ApoE proteins}

In order to compare the ZGO-PEG/protein interactions in a binary system, the capillary was preconditioned with mixtures of HSA and ApoE at different ratios, then ZGO-PEG were injected. Figure 4 shows a full panorama of these interactions. The electropherograms derived from the injection of ZGO-PEG in capillaries preconditioned with pure ApoE or HSA are presented in Figures $4 \mathrm{~A}$ and $4 \mathrm{~B}$, respectively, for the direct comparison of the effects derived from the addition of a second protein in the system. For evaluation of the cooperative interactions between ZGO-PEG and a binary system of proteins, the HSA and ApoE ratio was varied, with concentrations ranging from 0 to $0.72 \mu \mathrm{M}$ (Figures $4 \mathrm{C}$ and 4D). So as to facilitate the analysis of the results obtained from Figure 4, a schematic representation of the phenomena observed in these systems is presented in Figure 5.

In Figures $4 C$ and $4 D$, the superimposed dashed lines represent the injection of an ACB zone in the capillary pre-conditioned as indicated. These experiments demonstrate the inter-protein interactions between HSA and ApoE, evidenced by the formation of a positive broad peak (a plateau) with a mixture of proteins containing concentrations higher than $0.36 \mu \mathrm{M}$. As previously indicated in the literature, some peptides and more than thirty-five proteins can interact with HSA, including both high and low abundant proteins (e.g. apolipoproteins, angiotensinogen, ceruloplasmin, clusterin, hemoglobin ( $\mathrm{Hb})$, plasminogen, prothrombin, and transferrine). ${ }^{31,32}$

When the HSA concentration in the pre-conditioned capillary was fixed at $0.72 \mu \mathrm{M}$ and the ApoE was varied (Figure $4 \mathrm{C}$ ), a negative peak related to the HSA vacancy appears as previously explained but it is affected by the concentration of the ApoE. Since no significant variations in the area of the negative peaks were detected with low ApoE concentrations (ApoE $\leq 0.24 \mu \mathrm{M}$ ) compared to those observed for separations in a BGE with only $0.72 \mu \mathrm{M} \mathrm{HSA}$, it is deduced that HSA was not initially removed from the ZGO-PEG surfaces. However, when the ApoE concentration was increased (ApoE $\geq 0.36 \mu \mathrm{M}$ ), the equilibrium is modified and the vacancy peak area slightly diminished, indicating an increase in HSA concentration in the BGE, which could be due to a lower HSA adsorption at the ZGO-PEG surface.

According to Figures $4 \mathrm{~A}$ and $4 \mathrm{C}$, the electrophoretic mobility of the ZGO-PEG/proteins nanocomplex was significantly decreased (in absolute value) in the presence of a fixed $0.72 \mu \mathrm{M}$ HSA concentration with respect to experiments with only ApoE. A direct comparison of these values is presented in Figure 6. As described before, higher ApoE concentrations induce a gradual increase in the electrophoretic mobility (in absolute value) of the ZGO-PEG/ApoE nanocomplex due to the negative charges provided by the protein However, when a fixed concentration of HSA $(0.72 \mu \mathrm{M})$ is added in the $B G E$, this electrophoretic mobility (in absolute value) increases more slowly, probably due to the presence of HSA as well on NP surface. This indicates the formation of a ternary ZGOPEG/HSA/ApoE nanocomplex with slow dissociation kinetics, which could be assisted by the initial HSA/ApoE interprotein association. 

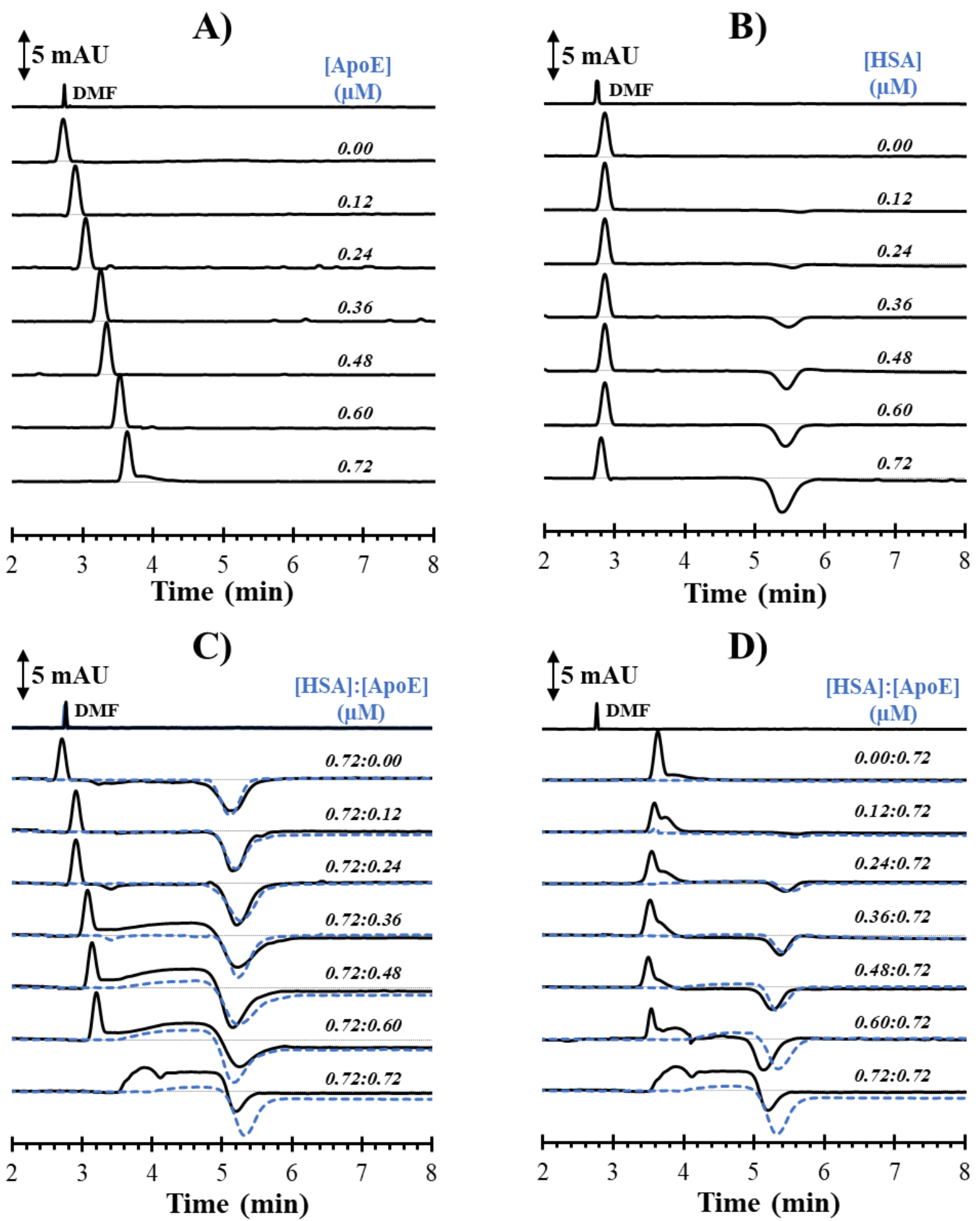

Figure 4. Electropherograms for evaluation of the cooperative interactions between ZGO-PEG and binary systems of proteins. a) interactions between ZGO-PEG and ApoE. b) interactions between ZGO-PEG and HSA. interactions between ZGO-PEG and a mixture of proteins at different rations: c) HSA concentration was fixed and ApoE varied, and d) ApoE concentration was fixed and HSA varied. in all the systems, the continuous lines correspond to the electropherograms obtained upon the ZGO-PEG injection, while the superimposed dashed lines in c) and d) correspond to the ACB injection. The indicated values are the final protein concentrations in the mixtures. The analyses were performed in $15 \mathrm{mM} \mathrm{ACB}(\mathrm{pH} 7.4)$. $\mathrm{E}=14.0 \mathrm{kV}$. 
Furthermore, when ApoE concentration in the mixture was $\geq 0.36$ $\mu \mathrm{M}$ (Figure 4C), a plateau appeared, connecting the ZGOPEG/proteins signal and the HSA vacancy peak, with higher intensity compared to those experiments in which only BGE was injected (dashed lines). This observation is in accordance with the formation of a ternary ZGO-PEG/proteins nanocomplex.

Figure $5 \mathrm{~B}$ schematizes the hypothetic complexes formed during this experiment: whereas the ZGO-PEG/HSA and ZGO-PEG/ApoE systems have fast and slow dissociation kinetics, respectively, the ternary ZGO-PEG/proteins nanocomplex satisfies two conditions: 1) it has a slow dissociation kinetics, and 2) it leads to the release of some HSA from the NP surface after a given ApoE concentration. At low ApoE concentrations, this ternary complex could exist with both HSA and ApoE proteins at the ZGO-PEG surface, unaffecting the vacancy peak area. Nevertheless, when increasing the ApoE concentration, some HSA could be released from the NP surface, which suggests the displacement of HSA by ApoE from the NPs surface through competitive interactions, triggering variations in the vacancy peak area. Due to the fast dissociation kinetics of the ZGO-PEG/HSA interaction, a ternary nanocomplex in which HSA connects the ZGO-PEG and the ApoE is not expected. As a slow dissociation nanocomplex was formed, its structure should correspond to a ternary ZGO-PEG/ApoE/HSA nanocomplex in which the ApoE acts as a bridge between the ZGO-PEG and the HSA.

In Figure 4D, in which the ApoE concentration was fixed at $0.72 \mu \mathrm{M}$ and the HSA concentration was varied, a peak associated with the ZGO-PEG/ApoE nanocomplex was registered in all the electropherograms at $3.6 \mathrm{~min}$ (the same time observed for the ZGO-PEG/ApoE nanocomplex formed in a capillary containing only $0.72 \mu \mathrm{M}$ ApoE). That migration time was kept constant when HSA concentration increased. However, a shoulder and then a peak deformation was evidenced as well as a broad peak between the HSA vacancy and the nanocomplex peak. These results provide further evidence of the ZGO-PEG/ApoE nanocomplex stability (slow dissociation kinetics), even in the presence of HSA, with the possible formation of the ternary ZGO-PEG/ApoE/HSA nanocomplex.

Globally, this set of experiments clearly demonstrates the stronger affinity of ZGO-PEG for ApoE compared to HSA, and the stability of the ZGO-PEG-ApoE nanocomplex, even in environments with higher HSA concentrations.

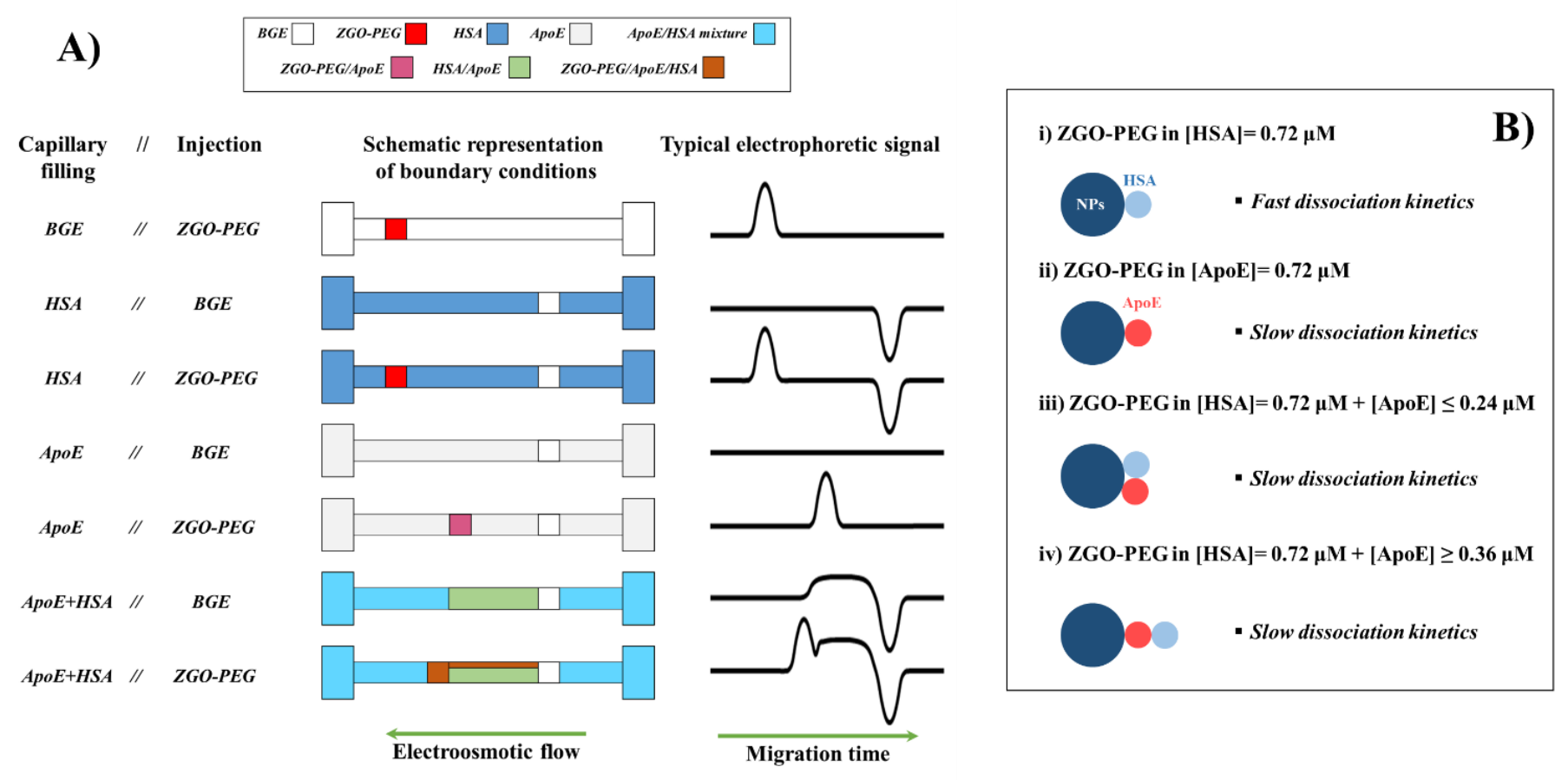

Figure 5. A) Schematic representation of interactions between ZGO-PEG and the binary system of HSA and ApoE proteins in a CE system. B) Hypothetic forms of the ZGO-PEG/binary-protein interactions. 


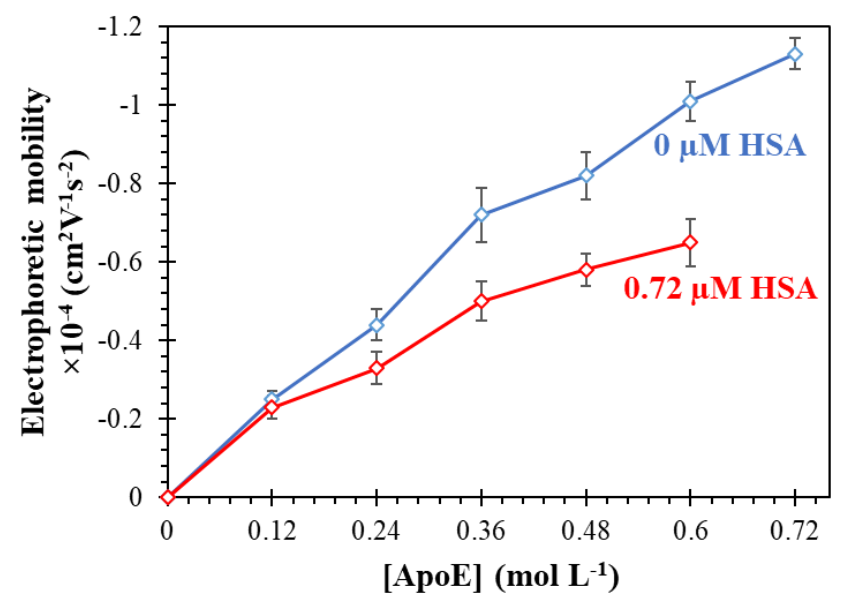

Figure 6. Effect of HSA $(0.72 \mu \mathrm{M})$ on the electrophoretic mobility of the ZGO-PEG/ApoE nanocomplex formed in a capillary preconditioned with ApoE at different concentrations, according to Figure 4C.

It furthermore evidences the HSA/ApoE association that seems to lead to the formation of a more complex interaction system involving ZGO-PEG and the two proteins through cooperative interactions, in which the most stable complex is formed with ApoE acting as a bridge between ZGO-PEG and HSA.

\section{Competitive interactions between ZGO-PEG and a mixture of the} two proteins: pre-incubation effects

In order to better understand the interaction mechanisms between ZGO-PEG, ApoE, and HSA in a competitive system model, different pre-incubation approaches were compared in various BGE compositions (Figure 7). The incubation of the ZGO-PEG with proteins was performed at the most elevated concentration analyzed in this work $(1.44 \mu \mathrm{M})$ in order to guarantee the NP saturation. Since no effect due to the incubation time was evidenced in the range from 15 minutes to 2 hours, the shortest time (15 minutes) was considered.

The characteristic peak of ZGO-PEG observed in Figure $7 a$ is a reference for the experiments described below. When HSA is present in the BGE (Figure 7b), a positive peak corresponding to ZGO-PEG and a peak with negative absorbance due to the vacancy of the HSA are present. When ApoE is present in the BGE (Figure 7c), the ZGO-PEG/ApoE nanocomplex was observed. The behavior of the ZGO-PEG in these two cases is the one described previously with individual proteins in the BGE. In Figure $7 d$, the incubated
ZGO-PEG with HSA is separated in a BGE composed of only ACB: two signals are observed, a positive peak with the same migration time of ZGO-PEG separated in a BGE containing HSA (Figure 7b), and a second one, positive as well, with the same migration time and area as for free HSA under same analytical conditions (See Figure S5). This indicates that the complex ZGO-PEG/HSA is not stable after a $15 \mathrm{~min}$ pre-incubation and separation under an electric field. When ZGO-PEG was pre-incubated with ApoE and separated in ACB (Figure 7e), the ZGO-PEG/ApoE nanocomplex appears at the same migration time of the nanocomplex in Figure $7 c$, in which NPs were injected in the capillary containing only ApoE. This indicates that a stable ZGO-PEG/ApoE nanocomplex was effectively obtained under pre-incubation. The protein interactions in a competitive system were then analyzed.

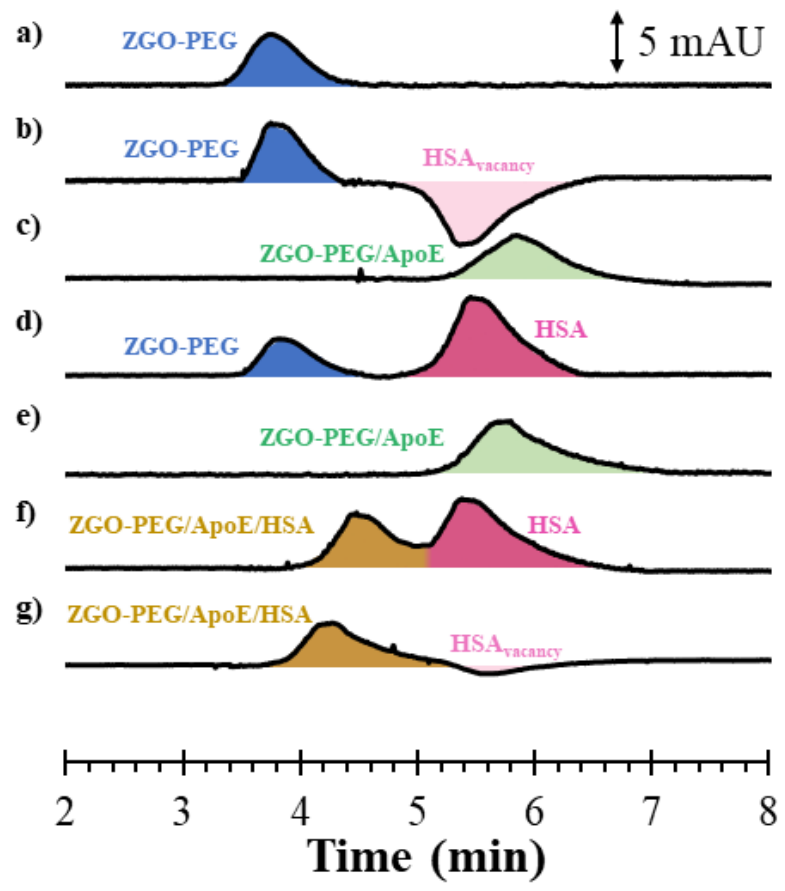

Figure 7. Set of representative electropherograms obtained at $\lambda=$ $200 \mathrm{~nm}$ for analysis of the competitive interactions. The electropherograms are identified according to the BGE composition (BGE) and pre-incubated sample (S): a) $B G E=A C B, S=Z G O-P E G, b$ ) $B G E=A C B+H S A, S=Z G O-P E G, \quad c) B G E=A C B+A p o E, S=Z G O-P E G, d)$ $B G E=A C B, S=Z G O-P E G / H S A$, e) $B G E=A C B, S=Z G O-P E G / A p o E, f)$ $B G E=A C B+A p o E ， S=Z G O-P E G / H S A ，$ g) BGE=ACB+HSA， S=ZGOPEG/ApoE. Protein concentration was $1.44 \mu \mathrm{M}$ for all cases. Initial BGE was $30 \mathrm{mM} \mathrm{ACB}$ (pH 7.4). E=12.0 kV. 
For this purpose, the ZGO-PEG was first incubated with HSA and then injected in a BGE containing ApoE, both of them at the same concentration $(1.44 \mu \mathrm{M})$. In Figure $7 f$, the peak of HSA was observed, with a smaller peak area compared to free HSA (Figure S5), while a second superimposed peak appears at an intermediary migration time between ZGO-PEG and the ZGO-PEG/ApoE nanocomplex. This indicates a competitive interaction between HSA and ApoE, in which the HSA is replaced by the ApoE at the ZGO-PEG surface due to its stronger interaction, and the formation of a ternary ZGOPEG/ApoE/HSA complex.

Finally, in Figure $7 \mathrm{~g}$ the pre-incubated ZGO-PEG and ApoE is separated in a BGE containing HSA. A very slight negative peak corresponding to the HSA vacancy is observed. Furthermore, a broad peak (or a peak followed by a plateau) is also present, at an electrophoretic mobility between the ones of ZGO-PEG in Figures $7 \mathrm{~d}$ and 7e. The broad peak is attributed to the ternary ZGOPEG/ApoE/HSA nanocomplex, which does not seem very stable. This is and additional evidence of the participation of HSA in the ternary nanocomplex. Accordingly, it is clearly demonstrated again that: (1) the ZGO-PEG/HSA complex is ruled by a fast association/dissociation kinetics, (2) the ZGO-PEG/ApoE is a stable nanocomplex, even in the presence of the competing HSA, (3) the ApoE is able to displace the HSA from the ZGO-PEG surface, and (4) an interaction between HSA and ApoE occurs, and a ternary bioconjugated nanocomplex ZGO-PEG/ApoE/HSA is formed under the analysis conditions. Considering the great stability of the ZGOPEG/ApoE nanocomplex, even in the presence of higher concentrations of other proteins such as HSA, the pre-formation of an ApoE protein corona around the ZGO-PEG by means of a simple 15 min pre-incubation step can be explored as a strategy for their translocation across the $\mathrm{BBB}$, representing a potential tool for optical imaging into the brain region. The set of methodologies presented herein can be potentially adapted to study other interacting NPs or specific proteins. This work also opens the perspectives for the evaluation of interaction between NPs and multiplex protein systems.

\section{Conclusions}

A systematic set of electrokinetic methodologies was implemented to deeply study the protein corona formation around PEGylated persistent luminescence NPs (ZGO-PEG). A binary system of proteins considering HSA, the most abundant serum protein, and ApoE, associated with the active transport of NPs through the blood-brain barrier, was analyzed. The stronger affinity between ZGO-PEG and ApoE compared to HSA was systematically evidenced through the application of comparative, cooperative, and competitive methodologies. Firstly, the interaction of nanoparticles with individual proteins in the capillary, and thereafter the comparison of the model fitting parameters, indicated binding constants $K_{a}=4.60 \pm 0.41 \times 10^{6} \mathrm{M}^{-1}$ and $K_{a}=1.96 \pm 0.25 \times 10^{10} \mathrm{M}^{-1}$ for the ZGO-PEG/HSA and ZGO-PEG/ApoE nanoconjugates, respectively in $30 \mathrm{mM}$ ammonium carbonate buffer $(\mathrm{pH}$ 7.4). The evaluation of the ZGO-PEG interactions with a mixture of these proteins at different ratios revealed three important parameters: the stronger interactions with ApoE, even in a system with high HSA concentration, the presence of HSA-ApoE inter-protein interactions, and the cooperative interactions allowing the formation of a ternary ZGO-PEG/ApoE-HSA nanocomplex. Finally, the competitive model showed dynamic NP-protein interactions in which the ApoE displaces the HSA from the ZGO-PEG surfaces. These CE characterizations demonstrated to be enough sensitive to detect small changes in the magnitude of association parameters. Even if the presence of only two proteins is far from the real protein composition in a physiological sample, at the current state of the research in which the interactions between nanoparticles and individual proteins is normally considered, the analysis of interactions in a binary system of proteins represents a great advance in the simulation of physiologically relevant conditions. This methodology could allow a better understanding of the interactions between nanoparticles and proteins, improving the development of novel functional nanomaterials for different biomedical applications like diagnostic biosensors, targeted therapy, and optical imaging. Furthermore, the Apo-E adsorption onto the ZGO-PEG induced by a fast incubation step could be explored as a simple modification strategy for their translocation across the blood-brain barrier, representing a potential tool for optical imaging into the brain zone.

\section{Supporting information}

See the online resources. 


\section{Author contributions (CRediT)}

Conceptualization-Ideas: GRG, FdO, AV; Formal analysis, investigation, methodology: GRG, FdO, AV; Project administration AV. Resources: GRG, CR, NM, FdO, AV; Supervision: AV; Validation: GRG, FdO, AV; Visualization: GRG; Writing-original draft: GRG; Writing, review and editing: GRG, FdO, CR, NM, AV.

\section{Conflicts of interest}

The authors declare no competing interests.

\section{Acknowledgements}

The authors extend their acknowledgments to Lourdes Palma Tirado (INB-UNAM) for her technical assistance during TEM observations. We also thank Pr. Karine Andrieux for helpful discussions regarding the interest of ApoE interactions with nanoparticles.

\section{References}

1 D. Maiolo, P. Del Pino, P. Metrangolo, W. J. Parak and F. Baldelli Bombelli, Nanomedicine, 2015, 10, 3231-3247.

2 Y. Sato, Y. Kinami, K. Hashiba and H. Harashima, J. Control. Release, 2020, 322, 217-226.

3 A. K. Mohammad and J. J. Reineke, Mol. Pharm., 2013, 10, 2183-2189.

4 T. Maldiney, M. Rémond, M. Bessodes, D. Scherman and C. Richard, J. Mater. Chem. B, 2015, 3, 4009-4016.

5 N. Hartl, F. Adams and O. M. Merkel, Adv. Ther., 2021, 4, 2000092.

6 M. Kristensen, D. Birch and H. Mørck Nielsen, Int. J. Mol. Sci., 2016, 17, 185.

7 S. Wagner, A. Zensi, S. L. Wien, S. E. Tschickardt, W. Maier, T. Vogel, F. Worek, C. U. Pietrzik, J. Kreuter and H. von Briesen, PLoS One, 2012, 7, e32568.

8 H. R. Kim, K. Andrieux, S. Gil, M. Taverna, H. Chacun, D. Desmaële, F. Taran, D. Georgin and P. Couvreur, Biomacromolecules, 2007, 8, 793-799.

9 S. Tenzer, D. Docter, J. Kuharev, A. Musyanovych, V. Fetz, R. Hecht, F. Schlenk, D. Fischer, K. Kiouptsi, C. Reinhardt, K. Landfester, H. Schild, M. Maskos, S. K. Knauer and R. H. Stauber, Nat. Nanotechnol., 2013, 8, 772-781.

10 T. Maldiney, A. Bessière, J. Seguin, E. Teston, S. K. Sharma, B. Viana, A. J. J. Bos, P. Dorenbos, M. Bessodes, D. Gourier, D. Scherman and C. Richard, Nat. Mater., 2014, 13, 418-426.

11 T. Lécuyer, E. Teston, G. Ramirez-Garcia, T. Maldiney, B. Viana, J. Seguin, N. Mignet, D. Scherman and C. Richard, Theranostics, 2016, 6, 2488-2523.

12 G. Ramírez-García, S. Gutiérrez-Granados, M. A. GallegosCorona, L. Palma-Tirado, F. d'Orlyé, A. Varenne, N. Mignet, C. Richard and M. Martínez-Alfaro, Int. J. Pharm., 2017, 532, 686695.

13 S. S. Aleksenko, A. Y. Shmykov, S. Oszwałdowski and A. R.
Timerbaev, Metallomics, 2012, 4, 1141-1148.

14 G. Ramírez-García, F. d'Orlyé, S. Gutiérrez-Granados, M. Martínez-Alfaro, N. Mignet, C. Richard and A. Varenne, Colloids Surfaces B Biointerfaces, 2017, 159, 437-444.

15 L. Trapiella-Alfonso, G. Ramírez-García, F. d'Orlyé and A. Varenne, TrAC Trends Anal. Chem., 2016, 84, 121-130.

16 G. Ramírez-García, L. Trapiella-Alfonso, F. d'Orlyé and A. Varenne, Capill. Electromigr. Sep. Methods, 2018, 397-421.

17 N. Li, S. Zeng, L. He and W. Zhong, Anal. Chem., 2010, 82, 74607466.

18 M. Matczuk, K. Anecka, F. Scaletti, L. Messori, B. K. Keppler, A. R. Timerbaev and M. Jarosz, Metallomics, 2015, 7, 1364-1370.

19 H. R. Kim, K. Andrieux, C. Delomenie, H. Chacun, M. Appel, D. Desmaele, F. Taran, D. Georgin, P. Couvreur and M. Taverna, Electrophoresis, 2007, 28, 2252-2261.

20 K. Andrieux and P. Couvreur, Wiley Interdiscip. Rev. Nanomed. Nanobiotechnol., 2009, 1, 463-474.

21 D. Brambilla, R. Verpillot, M. Taverna, L. De Kimpe, B. Le Droumaguet, J. Nicolas, M. Canovi, M. Gobbi, F. Mantegazza, M. Salmona, V. Nicolas, W. Scheper, P. Couvreur and K. Andrieux, Anal. Chem., 2010, 82, 10083-10089.

22 D. Brambilla, R. Verpillot, B. Le Droumaguet, J. Nicolas, M. Taverna, J. Kóňa, B. Lettiero, S. H. Hashemi, L. De Kimpe, M. Canovi, M. Gobbi, V. Nicolas, W. Scheper, S. M. Moghimi, I. Tvaroška, P. Couvreur and K. Andrieux, ACS Nano, 2012, 6, 5897-5908.

23 Y. François, K. Zhang, A. Varenne and P. Gareil, Anal. Chim. Acta, 2006, 562, 164-170.

24 T. Maldiney, C. Richard, J. Seguin, N. Wattier, M. Bessodes and D. Scherman, ACS Nano, 2011, 5, 854-862.

25 N. J. Anthis and G. M. Clore, Protein Sci., 2013, 22, 851-858.

26 T. R. C. Guizado, J. Mol. Model., 2014, 20, 2450.

27 Gref, Luck, Quellec, Marchand, Dellacherie, Harnisch, Blunk and Muller, Colloids Surf. B. Biointerfaces, 2000, 18, 301-313.

28 V. Kumar, J. Qin, Y. Jiang, R. G. Duncan, B. Brigham, S. Fishman, J. K. Nair, A. Akinc, S. A. Barros and P. V Kasperkovitz, Mol. Ther. Nucleic Acids, 2014, 3, e210.

29 M. T. Peracchia, S. Harnisch, H. Pinto-Alphandary, A. Gulik, J. C. Dedieu, D. Desmaële, J. d'Angelo, R. H. Müller and P. Couvreur, Biomaterials, 1999, 20, 1269-1275.

30 I. F. Tudorache, V. G. Trusca and A. V. Gafencu, Comput. Struct. Biotechnol. J., 2017, 15, 359-365.

31 G. Fanali, A. di Masi, V. Trezza, M. Marino, M. Fasano and P. Ascenzi, Mol. Aspects Med., 2012, 33, 209-290.

32 G. Rabbani and S. N. Ahn, Int. J. Biol. Macromol., 2019, 123, 979-990. 